\title{
A NOVEL PARADIGM SELECTION ALGORITHM IN OPPORTUNISTIC ROUTING
}

\author{
V.VALLINAYAGI 1 Dr.G.M.Nasira² \\ ${ }^{1}$ Research scholar, M.S University, Tirunelveli. \\ ${ }^{2}$ Chikkana Government College, Tirupur.
}

\section{Abstract}

Mainly opportunistic routing is for multihop wireless networks. Major issues on opportunistic routing is selection forwarding coordination. . In this paper we compare selected set of algorithm that has been proposed in literature. Generally routing protocols send traffic along reliable path and have some problem with path. Upon each transmission the candidate coordinate such that the most priority one receiving the packet will forward the data. So we propose an efficient energy, speed and link stability as selection criteria which take advantage of the geographic routing and broadcast nature of wireless medium. Forwarding forms the basic criteria for sending the data in opportunistic routing. In this paper we compare candidate selection algorithm with the proposed algorithm. To avoid packet duplication, the first node that is selected suppresses the selection of further nodes. Since the basic scheme can lead to packet duplication, we describe alternative ways of suppressing those. The proposed method will consume more energy and accommodating more hosts. We finally give common approach for forwarding packets.

Keywords: Manet, Candidate Selection, opportunistic routing, performance, extremely opportunistic routing, novelty, packet forwarding, least cost opportunistic routing.

\section{INTRODUCTION}

A network consists of host that communicates without a fixed infra structure. Each hub has the ability to communicate with other nodes over a wireless channel. Every host has to determine its environment when the network is formed. Let us assume each node has a power global position system receive which make us to know the position of the node itself. On the unavailability of GPS we measure the distance of adjacent nodes with the help of incoming strength. MANETs are characterized by the mobility of nodes, which can move in any direction and at any speed that may lead to arbitrary topology and frequent partition in the network. This characteristic of the network makes the development of routing protocols as one of the most challenging issue.

Routing is the major task of the network. In routing mobility of the nodes are measured. Most probably nodes require the position itself and one hop neighbor. Consequently nearest nodes are aware of distance between them. Existing network layer protocols for adhoc networks[1] assume an ideal physical layer model where two nodes communicate if and only if the distance between them is at most is $\mathrm{R}$. Within transmission medium can exchange correctly bits. The probability of receiving successful depends on the probability of receiving bits successfully. In this paper we consider the routing with acknowledgment and choose hop by hop transmission. A Packet is retransmitted between nodes until it is received and acknowledged correctly.

When a source node wants to transmit a packet it gets the location of the destination first and attach it to packet header. Due to the Destination node's movement the multihop path may diverge from the true location of the final destination and a packet would be dropped even if it has already been delivered into the neighbourhood of the destination. Forwarding is nothing but an area specified for selecting but an area specified for selected hops for sending information from source to destination [2]. The basic routing scheme of POR Can be discussed in fig1. In normal situation without link break. The packet is forwarded by the next hop node and forwarding neighboring hops are all selected with highest priority. Here we use selection and prioritization of forwarding neighboring hops.

There are three main forwarding strategies used for position based protocol greedy forwarding, restricted forwarding and hierarchical approaches. Most position based protocols use greedy forwarding to route packets from source to destination. Most position based protocols use greedy forwarding to route packets from source to 
destination. We presume that all nodes broadcast with equal transmission power. Therefore all nodes have equal and fixed conduction radius. $A$ sender node has a packet that it wishes to deliver to a distant destination. Now the sender node sends the packet by means of broadcasting. A subset of nodes receives the packet, Now it is the duty of a protocol to decide which nodes should be in the subset. The way of broadcasting is by the neighbouring nodes near the sender. The node which is closest will broadcast the packet and also check node should lie towards the destination. This process continues until it reaches destination. In this paper we consider with acknowledgement in the hop by hop retransmission model. A packet is retransmitted between two nodes until it is received and acknowledged correctly. A direct communication between two units is called a single hop communication, while the communication is said multihop when a unit must rely on other units to forward its messages. A multihop communication is composed of several single hop communications one after another. There are two important issues in opportunistic routing are candidate selection and priority assignment. In this paper, a novel Position based opportunistic routing protocol is Proposed in which several forwarding candidate nodes are selected with criteria basis.

The main contribution of this paper is

- We propose position based opportunistic routing mechanism which the nodes organize without multifarious adjustment to MAC protocol.

- First the selection and prioritization of forwarding is done through candidate selection algorithm.

- We propose criteria like energy, speed and link stability which is calculated for each node in the selection area and select the best forwarder among the chosen node.

- We compare candidate selection algorithm with proposed algorithm.

- Finally simulation performance was investigated and verify the performance when the path is created for sending data.

\section{A. Candidate selection algorithm:}

Selection of nodes inside the forwarding area get the chance to catch the message to next hop. A node located in forwarding area satisfy the following two Conditions It makes positive progress towards destination. It 's distance to next reappears to be more difficult than routing itself. hop node should not exceed half of the transmission of wireless nodes so that ideally all forwarding candidate can hear from one another. Candidate selection Algorithm:[4]

Algorithm 1 shows the procedure to select and priority forwarder list.

List N : Neighbour list

List C : Candidate list, initialized as empty list.

$N_{D}$ : Destination node

Base : Distance between current Node and $\mathrm{N}_{D}$

If Find (list $N, N_{D}$ ) then Next_hop

Return ; End if

For $\mathrm{p} \leftarrow-0$ to length(list N) do

List N[i] dist $\leftarrow$-dist $\quad\left(\right.$ List N[i]. $\left.N_{D}\right)$

End for

List N.sort()

Next hop $\leftarrow$ List $\mathrm{N}[0]$

For $\mathrm{i} \leftarrow 1$ to length (Liat N) do

If dist ( List $\left.\mathrm{N}[i], \mathrm{N}_{D}\right)>=$ base or Length

$($ List $\mathrm{C})=\mathrm{N}$

Then Break

\section{Else if}

Dist(List $\mathrm{N}[\mathrm{i}]$,listN[0]< $\mathrm{R} / 2$ then

List C.add (List N[i]) ;

endif; endif;

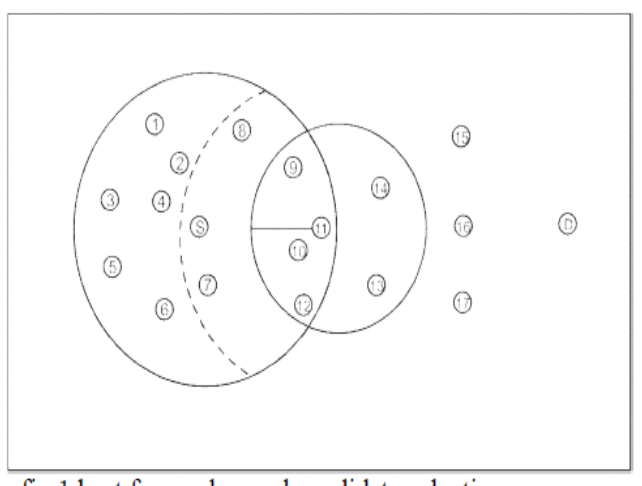

fig 1 best forwarder and candidate selection

In this algorithm only the nodes in the forwarding area get the chance to forward the packets. The selection area is determined by the sender and the next hop node. Here an array is maintained. The priority of the forwarding nodes are measured by the distance. The distance measured between the source node and destination node first, then selection is made to the neighbouring nodes. Sender node first took all the neighbouring nodes and 
measure the distance between the destination node and neighbouring nodes which lie in front of the source node. The distance is calculated and arranged in the array. The nearer to the destination is given higher priority for forwarding the node, it gets the first preference to forward the next hop towards the destination. The candidate list is attached to the packet header a forwarding candidates. And update hop by hop. Only the nodes specified in the list will be forwarded.

\section{B. Extremely opportunistic Routing(ExOR)}

Exor[11] uses ETX as the metric for selected candidates algorithm. It runs on shortest path first with weight. The first node after the source in this path is selected as candidate ETX Is used to sort the candidate set. Then the link between $s$ and $c$ is removed and the loop is repeated until no more paths to $d$ available or maximum number of candidates is reached.

Algorithm 2. Candidate selection ExOR(s,d,ncand)

1: Gtmp = temporal copy of the network topology

2: $\operatorname{cost}(\mathrm{s}) \mathrm{ETX}(\mathrm{s}, \mathrm{d})$ in Gtmp; Cs, d ;

3: while jCs,dj < ncand \& (s,d) connected in Gtmp do

4: cand first node after $s$ in the $\operatorname{SPF}(\mathrm{s}, \mathrm{d})$ in $\mathrm{Gtmp}$

5: if cand $==d$ then

6: Cs,d Cs,d [ $\{\mathrm{d}\}$

7: $\operatorname{cost}$ (cand) 0

8: else

9: cost(cand) ETX(cand,d) in Gtmp

10: if cost(cand) < cost(s) then

11: Cs,d Cs,d [ \{cand\}

12: end if

13: end if

14: Gtmp delete edge(s,cand) in Gtmp

15: end while

16: Cs,d Cs,d ordered by cost.

\section{LCOR[12]}

The goal of this algorithm is to find the optimal candidates sets. Recall that the optimal candidates sets are the sets that minimize the expected number of transmissions from source to the destination. It works similar to the classical distributed Bellman-Ford algorithm.

Algorithm 3. Candidate selection LCOR(s,d,ncand) 1: for all $v$ in the network $n\{d\} d o$
2: costcurr(v) 1; $\operatorname{costprev(v)~} 1$

3: end for

4: costcurr(d) 0

5: repeat

6: flag TRUE

$7:$ for all $v$ in the network $n\{d\}\{$ search for the best candidates set\}

8: Cv;d arg minS22jNðvpj ;0<jSj6ncandEAXðS; v; dp

9: costcurr(v) $\operatorname{EAX}(\mathrm{Cv}, \mathrm{d}, \mathrm{v}, \mathrm{d})$

10: end for

11: for all $v$ in the network $n\{d\}$ do

12: if costcurr(v) - costprev(v) then

13: costprev(v) costcurr(v)

14: flag FALSE

15: end if

16: end for

17: until flag $==$ TRUE

18: Cs,d Cs,d ordered by costcurr

\section{RELATED WORK}

Hightower proposed a position based approach in routing becomes practical due to the rapidly developing software and hardware solutions for determining absolute or relative positions of nodes in indoor/outdoor ad hoc networks.

Stojmenovic addressed the problem of designing location update schemes to provide accurate destination information and enable efficient routing in mobile ad hoc networks a

In, Zhong [12]et al. proposed a new metricexpected any-path transmission (EAX) -that generalizes ETX to an OR framework and proposed a candidate selection and prioritization scheme based on the new metric. They analyzed the efficacy of OR by using this metric and did a comparison using the link-level measurement trace of MIT Roofnet. In a distributed algorithm for computing minimum cost opportunistic routes, which is a generalization of the well-known Bellman-Ford algorithm, is presented.

Zubow[8] et al. in claimed that shadow fading losses for spatially close candidates are not independent from each other, unlike commonly assumed. They presented measurements obtained from an indoor testbed and concluded that correlations cannot be neglected if nodes are separated by less than $2 \mathrm{~m}$. 


\section{PROPOSED ROUTING PROTOCOLS}

(Node density, Energy and link stability) NDEL-POR based opportunistic protocols is designed to achieve maximum reliability in a mobile network. It combines geographical and opportunistic to achieve the high packet delivery ratio. The protocol chooses the best forwarder based on the energy, speed and link stability, when the best forwarder fails the next suboptimal forwarder will chose the node to forward. For transmitting the data packets from one source node (S) to another destination node (D), many transmission paths may be available with varying delay parameters involved. So as to send the data packet in least time period, the routing protocols often uses the minimum time utilization path between $S$ and $\mathrm{D}$ node. This path is known as the Best Path. While choosing the best path, the routing protocols usually neglect the current power levels of the nodes in the path. The relationship between transmit speed and overall energy consumption is complex. Reduced data transmit and receive times have only limited impact on per-packet energy consumption, due to the high fixed overhead. The main purpose of energy efficient algorithm is to maximize the network lifetime.

\section{A. Architectural design}

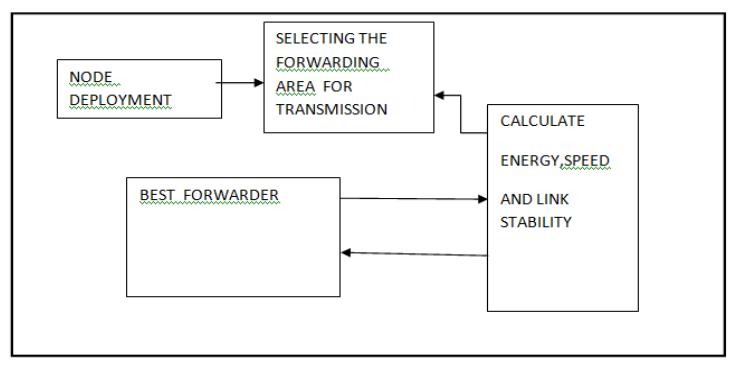

Fig 2 Architecture of NDEL-POR

Fig 2 shows the architecture of design of NDEL-POR For randomly deployment of nodes energy, speed and link stability [7] metric calculation facilitate the best forwarder and candidate selection. Best forwarder 1 and best forwarder 2 is chosen according to the metrics.

\section{B. Distance calculation and other metrics}

It is based on Euclidean distance

$$
\mathrm{d}=\sqrt{(\mathrm{x} 1-\mathrm{x} 2)^{2}+(\mathrm{y} 1-\mathrm{y} 2)^{2}}
$$

where $\mathrm{x} 1$ and $\mathrm{x} 2$ are the $\mathrm{x}$ coordinates and $\mathrm{y} 1$ and $\mathrm{y} 2$ are the $y$ coordinates.
Energy: Its energy is calculated Energylevel= metric1*0.5+(0.5*\$linksta(\$cnode,\$bestf1 $))$

Speed: Speed $=\left[0.5^{*}(100-\right.$-nodespeed]where energy is calculated by number of neighbors and their energy level and speed of each node is calculated. Forwarding area[10] is selected as the intersection area of the transmission range of the source node and half of the transmission range of the best forwarder. Among the nodes within this intersection area only those nodes which are closer to the destination than the source. The candidate list is maintained. Nodes expend energy while sending and receiving the packets. In node mobility information about node neighbors are always up-todate. Consider a particular forwarder $f_{i}$ as an Ex time elapsed Can be $S_{t}=t \quad r_{i}(t, s)=v . t \quad$ According to the transmission range energy is considered and speed of the node is also extended.Packet forwarding will fail due to node mobility and speed. We calculate from 100 as initial state and node up-to-date level will reduce from 100. Path length is seen for high energy and speed nodes with their neighbors. So the node first analyzes the sender node to the neighbouring node in time. Totally all the nodes get the message and their time is calculated. Then Message Successive Rate with link stability is investigated for all the neighboring nodes. Now the sender send the packets, in the first iteration nodes which are all neighbors are brought into focus. each neighbouring node with energy level, number of neighbors Are measured and investigate the link stability With these measure value the highest metric (speed, energy and link stability) is chosen as best forwarder.

\section{FORWARDING NODE SELECTION}

In fig2 node $S$ is the source node and $D$ is the destination node $R$ is the radius of the transmission range of $S$ is denoted by the circle as dotted lines. The node in the area enclosed within the dashed line and make positive progress towards the destination. From these nodes the one with maximum energy, speed and link stability is calculated as the best forwarder is chosen.

\section{Algorithm 1: Best Forwarder and Candidate Nodes Selection}

step1. Find if Destination node is in the Neighbour List. 
step2. If found, set the next hop as Destination node and exit. Else continue.

step3. For each node in the Neighbour List, do the following:

step3.a. Check if its distance from the Destination node is greater than or equal to the distance between the current node and Destination node.

If yes, break. Else, add node to an array.

Step4. Calculate the METRIC VALUE for all the nodes in the array Metric $=W_{\text {energ }}+W_{\text {speed }}+W_{\text {Istab }}$

Step5. Choose the node having the maximum metric value as the Best Forwarder.

In this paper the nodes are checked with energy, its speed link stability together they are taken priority and hen selection is made. Now we are ready to verify our claim that a node may not choose all its neighbors into the forwarder list as the optimum forwarder list at the beginning of this section. Each node in the network observes the behavior and information of its neighbors. It observes mobility of nodes; number of packets generated and number of nodes forwarded by the neighboring nodes and listen the past activity of the node.

\section{COMPARATIVE ANALYSIS}

We compare the performance of each algorithm in terms of the expected number of transmission needed to and a packet from the source to the destination and execution time of each algorithm. Regarding expected number of transmission LCOR Chose the same candidate set for transmission. EXOR is larger than any other OR algorithm.

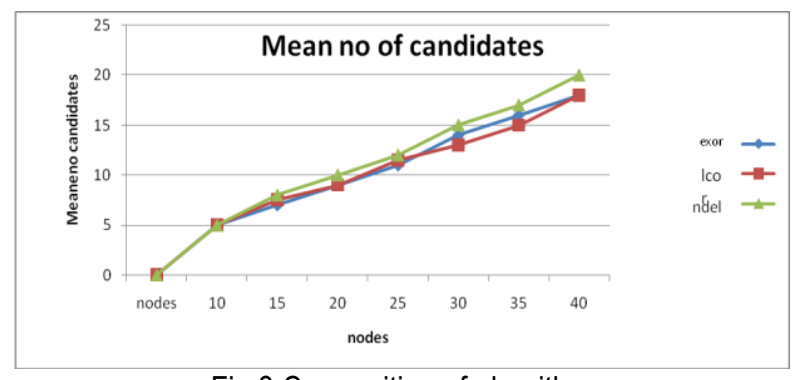

Fig.3 Comparition of algorithm

Exor is the simple algorithm that looks for the candidate runing and close to each other. So the investigation the optimal position of the candidates. But distributed due to the destination. Therefore the NDEL act as the coarse selection of the candidate list. Thus the expected number of tranmission is obtained for ncand $=1$ is same for all. Since all the algorithm has the same cadidnate set. EXOR forms for dense network. Thus limiting more candidates makes the selection more significant. but when we use NDEL We select the best forwarder in dense network without any complexity.

Table 1: Comparison Table for Candidate selection Algorithm

\begin{tabular}{|c|c|c|c|}
\hline & EXOR & LCOR & NDEL \\
\hline Candidate set & Same larger & Same medium & Same larger \\
\hline $\begin{array}{l}\text { Expected } \\
\text { transmission time }\end{array}$ & $\begin{array}{l}\text { Guarantee for } \\
\text { choosing optimum } \\
\text { set }\end{array}$ & Chosing a set & $\begin{array}{l}\text { Refiable chosing } \\
\text { the set }\end{array}$ \\
\hline Dense area & $\begin{array}{l}\text { Higher in dense } \\
\text { area }\end{array}$ & $\begin{array}{l}\text { Medium in dense } \\
\text { area }\end{array}$ & Same as exor \\
\hline Execution time & $\begin{array}{l}\text { Longest time for } \\
\text { execution }\end{array}$ & Longest time & $\begin{array}{l}\text { Time is less during } \\
\text { execution }\end{array}$ \\
\hline network & $\begin{array}{l}\text { Dynamic } \\
\text { computational } \\
\text { simplicity }\end{array}$ & $\begin{array}{l}\text { High } \\
\text { computationa! }\end{array}$ & $\begin{array}{l}\text { Dynamic network } \\
\text { simplicity }\end{array}$ \\
\hline $\begin{array}{l}\text { Time for sending } \\
\text { the packets }\end{array}$ & maximum & minimum & bow \\
\hline
\end{tabular}

We evaluate the computational cost of the algorithm by measuring the execution time. These Measures obtained having 10 counts. For all the algorithm the larger is the number of nodes the lower is the expected number of times. Exor is fastest and Lcor is slowest. Ndel protocol is a fastest when compared with other protocols. It takes less time to send the packet. Suppression if it does not have energy, speed and link stability. So Area coverage is less. By comparing the three algorithm NDEI protocol is fastest and execution time will also be less.

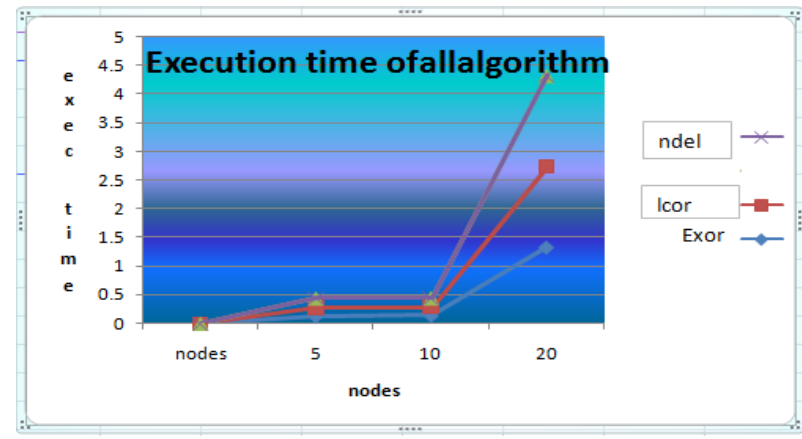

Fig.4 Execution time of all algorithm.

\section{CONCLUSION}

In this paper we have compare two relevant candidate algorithm with proposed algorithm. Relentlessly 
changing network topology makes predictable changing network performance with acceptable performance. The advantage of position-based routing over other ad-hoc routing protocols is the fact that nodes require only knowledge about the local neighborhood and the destination's location instead of global route topology. The node density, energy and link stability is proposed for routing in Manet. Inspirational by opportunistic routing, we propose a Novel position based routing protocol which takes benefit of stateless chattels of geographical routing and relay nature of wireless medium. Results of all the algorithm for number of transmission and Execution time to send the data is also compared. NDEL algorithm execute less nodes and time is also less when compare to EXor and Icor.

NDEL reassurance through best forwarder selection based on the node's link quality. Still the hops can be reduced by finding a new frame work for forwarding area. So that number nodes can be scrutinized and time complexity maybe less.

\section{REFERENCE}

[1] A Trivino Cabrera s. Canada-hurtado "Survey on Opportunistic Routing in multihop wireless network" information security (IJCNIS)vol.3 August2011

[2] Ana Maria popescu lon ,A.Hkemp " surveying position based Routing protocols foe wireless adhoc network"International communication networks and information security.vol 4,no 1 April2012.

[3] H.Takagi I.Kleinrock " optimal Tranmission ranges for randomly distributed packet radio terminals", IEEE Transactions on communication vol 32 no 31984 pp 246257.
[4] Shengb Yang Chai Kiat Yeo and Bu Sung Lee. "Toward Reliable data Delivery for highly Dynamic Mobile Adhoc networks",IEEE Transaction on mobile computing ,vol .11,No 1 Jan 2012.

[5] Dazhi Chen, Jing Deng, and Pramod K. Varshney, "Selection of a Forwarding Area for Contention-Based Geographic Forwarding in Wireless Multi-Hop Networks", IEEE Transactions on vehicular technology, vol. 56, no. 5, 2007.

[6] Z. Zhong, J. Wang, S. Nelakuditi, G.-H. Lu, On selection of candidates for opportunistic anypath forwarding, SIGMOBILE Mob. Comput.Commun. Rev. 10 (4) (2006) 12.

[7] Sahaya Rose Vigita, E., \& Golden Julie, E. (2013). Reliable Link-Based Routing Protocol for Highly Dynamic Mobile Adhoc Networks. International Journal of Engineering Trends and Technology (IJETT), 4(5), 1636-1642.

[8] Anupriya, A., \& Jubin, S. E. (2014). A study of efficient anonymous routing protocols in MANET. International Journal of Computer Applications (0975 - 8887), 91(8).

[9] Zeng, K., Yang, Z., \& Lou, W. (2009). Location-Aided Opportunistic Forwarding in Multi-Rate and Multi-Hop Wireless Networks. IEEE Transactions On Vehicular Technology, 58(6), 3032-3040.

[10] Rozner, E., Seshadri, J. , Mehta, Y. Lili Qiu, "SOAR: Simple Opportunistic Adaptive Routing Protocol for Wireless Mesh Networks", IEEE Transactions on Mobile Computing,Volume: 8 , Issue: 12, Page(s): 1622 - 1635 , Dec. 2009.

[11] Sanjit Biswas, Robert Morris, "ExOR: opportunistic multihop routing for wireless etworks", Proceedings of the 2005 conference on Applications, technologies, architectures, and protocols for mputer communications, Pages 133-144,2005.

[12] H.Dobius Ferrier ,M.grossglauser Least cost Opportunistic Routing In proceedings of 2007 Allerton conference on communication control and communicating. 